\title{
The Evolving Role of Rituximab in Adult Minimal Change Glomerulopathy
}

\author{
Landon C. Brown ${ }^{a}$ Meghan A. Jobson ${ }^{a}$ b Fernanda Payan Schober ${ }^{b}$ \\ Emily H. Chang ${ }^{b}$ Ronald J. Falk ${ }^{a, b}$ Patrick H. Nachman ${ }^{a, b}$ \\ William F. Pendergraft ${ }^{a, b}$ \\ a University of North Carolina School of Medicine, and ${ }^{b}$ University of North Carolina Kidney Center, \\ Division of Nephrology, Department of Medicine, Chapel Hill, NC, USA
}

\section{Keywords}

Minimal change glomerulopathy $\cdot$ Rituximab · Outcomes

\begin{abstract}
Background: Minimal-change glomerulopathy is defined histologically by the presence of normal glomeruli on light microscopy and diffuse podocyte effacement on electron microscopy. Although effective in children, corticosteroid treatment in adults is more variable and time to response can be prolonged. Data to support rituximab use in adults with corticosteroid-dependent or resistant minimal-change glomerulopathy are limited. Here, we describe the clinical course of adults with corticosteroid-dependent or -resistant minimal-change glomerulopathy who received rituximab. Methods: Demographic and clinical data were collected and analyzed from all adult patients with native kidney, biopsyproven, minimal-change glomerulopathy who were administered rituximab between 2009 and 2014 and cared for at the UNC Kidney Center. Results: Ten patients with corticosteroid-resistant $(n=5)$ or corticosteroid-dependent $(n=5)$ idiopathic minimal-change glomerulopathy were treated with rituximab between 2009 and 2014. Rituximab treatment induced remission in all 10 patients with a median time to remission of 2 months. The median time from rituximab to corticosteroid discontinuation was 3.5 months. The me-
\end{abstract}

dian remission time was 29 months and follow-up time was 39.5 months. No serious adverse events attributable to rituximab were observed. Conclusion: Rituximab induced remission in all patients with corticosteroid-dependent or -resistant minimal-change glomerulopathy, and may hold great therapeutic potential with good efficacy and minimal toxicity. Mounting evidence implies that a well-conducted randomized controlled clinical trial using rituximab in adults with minimal-change glomerulopathy in both corticosteroid-resistant and corticosteroid-dependent patients is warranted.

(c) 2017 S. Karger AG, Basel

\section{Introduction}

Minimal-change glomerulopathy, which is a clinicopathologic diagnosis made in patients who usually present with the nephrotic syndrome, is defined histologically by the presence of normal glomeruli on light microscopy and diffuse podocyte effacement on electron microscopy. In children, the disease is notably corticosteroid responsive, with over $85 \%$ of treated patients achiev-

L.C.B. and M.A.J. contributed equally to this work.

\section{KARGER}

(c) 2017 S. Karger AG, Basel

E-Mail karger@karger.com

www.karger.com/ajn
Dr. Will Pendergraft III

UNC Kidney Center, Division of Nephrology

Department of Medicine

Chapel Hill, NC 27599-7155 (USA)

E-Mail will_pendergraft@med.unc.edu 
ing complete remission within 8 weeks of treatment. Treatment with corticosteroids alone is less effective in adults, and with increasing age there is less likelihood of achieving complete remission. Response rates can be improved by extending the treatment duration, but the subsequent clinical course is complicated by frequent relapses requiring repeated treatment courses [1-6]. While spontaneous remission is rare in adults, complications of nephrotic syndrome are significant; therefore, successful treatment is imperative [7-9]. Given the frequent failure of corticosteroids to induce long-term remission and the toxicity associated with prolonged or repeated steroid exposure, a variety of therapies, including cyclophosphamide, calcineurin inhibitors, and mycophenolate mofetil, have been investigated as alternative treatment options in the setting of frequently relapsing, corticosteroid-dependent or corticosteroid-resistant disease. Unfortunately, the quality of the data to support the use of any of these agents is poor, with tacrolimus, for example, resulting in chronic nephrotoxicity [10]; thus, more effective treatment methods are needed [11].

Recently, the humanized murine monoclonal antibody rituximab, directed against CD20 located on the surface of B lymphocytes, has shown promise as a potential corticosteroid-sparing agent in frequently relapsing or corticosteroid-dependent minimal-change glomerulopathy. While use of this agent has predominantly been explored in pediatric populations [12-18], data suggesting effectiveness in adult patients are emerging. Studies in adult populations have largely been restricted to centers outside the United States (US) [19-25], and have focused on patients with frequently relapsing or corticosteroid-dependent disease rather than corticosteroid-resistant disease. Rituximab treatment for patients with corticosteroid-resistant disease has been described as successful in 2 children with minimal-change glomerulopathy [26], 2 case reports in adults $[27,28]$ and in a cohort study that included one resistant patient [19]. There is a dearth of information regarding rituximab treatment in corticosteroid-resistant adults and there is no reported comparison of patients with corticosteroid-dependent and -resistant disease treated with rituximab. Data reporting efficacy of rituximab in adult patients with corticosteroid-dependent or -resistant minimal-change glomerulopathy in a US setting, which includes patients with African ancestry are lacking.

In this report, we describe the clinical course of 10 adult patients with corticosteroid-dependent or -resistant minimal-change glomerulopathy treated with rituximab at our institution.

\section{Methods}

\section{Study Population}

All adult patients 18 years of age or older who were cared for at the UNC Kidney Center and who were administered an initial dose of rituximab between 2009 and 2014, for treatment of corticosteroid-dependent or -resistant biopsy-proven minimalchange glomerulopathy were considered for study inclusion. Exclusion criteria included either prior rituximab therapy or minimal-change disease that was deemed to be secondary to another process (malignancies, drug culprits or infections). The UNC Institutional Review Board approved this study (IRB 97-0523).

\section{Data Source}

Consecutive patients were identified by reviewing the electronic charts of patients at the UNC Kidney Center with native kidney, biopsy-proven, minimal-change glomerulopathy, including those who had received rituximab. Data regarding baseline demographic, clinical, laboratory, prior treatment characteristics and adverse events were extracted from patients' electronic medical records.

\section{Treatment}

All patients received either 2 doses of $1,000 \mathrm{mg}$ spaced 2 to 3 weeks apart (2 dose regimen) or 4 doses of $375 \mathrm{mg} / \mathrm{m}^{2}$ spaced 1 week apart (4 dose regimen) of intravenous (IV) rituximab. Patients re-treated with rituximab were given 1 or 2 doses of 1,000 $\mathrm{mg}$. Concomitant immunosuppression was tapered in all patients. Patients were premedicated with $125 \mathrm{mg}$ of methylprednisolone for all infusions. Of note, PJP prophylaxis was not used for patients receiving rituximab; however, proton pump inhibitors were used for all patients receiving corticosteroids.

\section{Clinical Assessment, Definitions, Measurements,}

\section{and Outcomes}

Corticosteroid-dependent disease was defined as relapse occurring during steroid tapering or within 2 weeks of steroid discontinuation. Corticosteroid-resistant disease was defined as unresponsiveness to at least 8 weeks of steroid therapy. Patients were evaluated at the UNC Kidney Center in our glomerulonephritis clinic within the first 3 months after rituximab therapy, and at 3-6 month intervals thereafter, at which time they were evaluated for symptoms and/or signs of remission as well as for adverse events. Serum albumin, serum creatinine, peripheral blood CD19/20-positive B cells, urinalysis, and urine protein to creatinine ratio (UPC) were measured at these time points and at times of possible disease flare. Remission was defined as a UPC $<0.3$ or negative urinalysis for protein and if available, albumin $>3.5 \mathrm{~g} / \mathrm{dL}$. Relapse was defined as a UPC $>3.5$ in a patient previously in remission.

\section{Statistical Analysis}

Baseline characteristics are presented individually for each patient, and summarized using frequency (\%) for categorical variables and median (range) for continuous variables. Laboratory values at the time of rituximab treatment, and monthly thereafter (when available) until last follow-up, are presented graphically. Statistical analyses were computed and figures were created using GraphPad Prism 5.0. 


\section{Results}

\section{Clinical Characteristics}

We identified 10 patients with either corticosteroidresistant $(n=5)$ or corticosteroid-dependent $(n=5)$ idiopathic minimal-change glomerulopathy treated with rituximab at our center between 2009 and 2016. Baseline demographic and clinical characteristics are presented in Table 1 . The median age at the time of rituximab administration was 54 (range 20-78) years. Men comprised $80 \%$ of the group and $70 \%$ of the patients were white. Baseline laboratory parameters were consistent with those of the nephrotic syndrome with a median UPC of 6.5 (range 0.44-15.75) and median serum albumin 2.5 (range 1.94). Three patients had evidence of impaired renal clearance at diagnosis and the remaining patients had a mean serum creatinine of $0.94 \mathrm{mg} / \mathrm{dL}$.

All patients were receiving corticosteroids at the time of rituximab administration and all had prior or current exposure to at least one additional immunomodulating agent (Table 1). All corticosteroid-dependent patients were prescribed steroids for at least 1 year or more prior to rituximab treatment, some for as long as 25 years continuously.

\section{Treatment and Outcomes}

Eight patients received the 2-dose regimen of rituximab and 2 patients received the 4 -dose regimen (Table 1 ). Subsequent treatment for relapse or maintenance was utilized in 4 patients. Individual patient information is listed in Table 2. Median follow-up time in the cohort overall was 40 months (range 20-80).

All 10 patients reached remission (defined by proteinuria $<0.3 \mathrm{~g}$, albumin $>3.5 \mathrm{~g} / \mathrm{dL}$, and normalized creatinine) and median time from rituximab administration to remission was 2 months (range 1-32, Table 2; Fig. 1). Of note, patient 7 , who was corticosteroid-resistant, achieved complete remission at the 18 months mark and required one additional dose of rituximab at the 22 months mark because she flared but had since maintained durable remission for over 2 years. The remaining 9 patients all achieved complete remission with a single-remission, induction regimen of rituximab. Median albumin after remission was $4.1 \mathrm{~g} / \mathrm{dL}$ (3.7-4.5) and median creatinine after remission was $0.88 \mathrm{mg} / \mathrm{dL}(0.5-1.5)$ for all 10 patients. Nine patients had discontinued corticosteroid therapy after a median time of 3.5 months. One patient (number 7) did not achieve remission after her first rituximab treatment; her peripheral B cells repopulated 3 months after rituximab treatment and alternative treatments were pur-

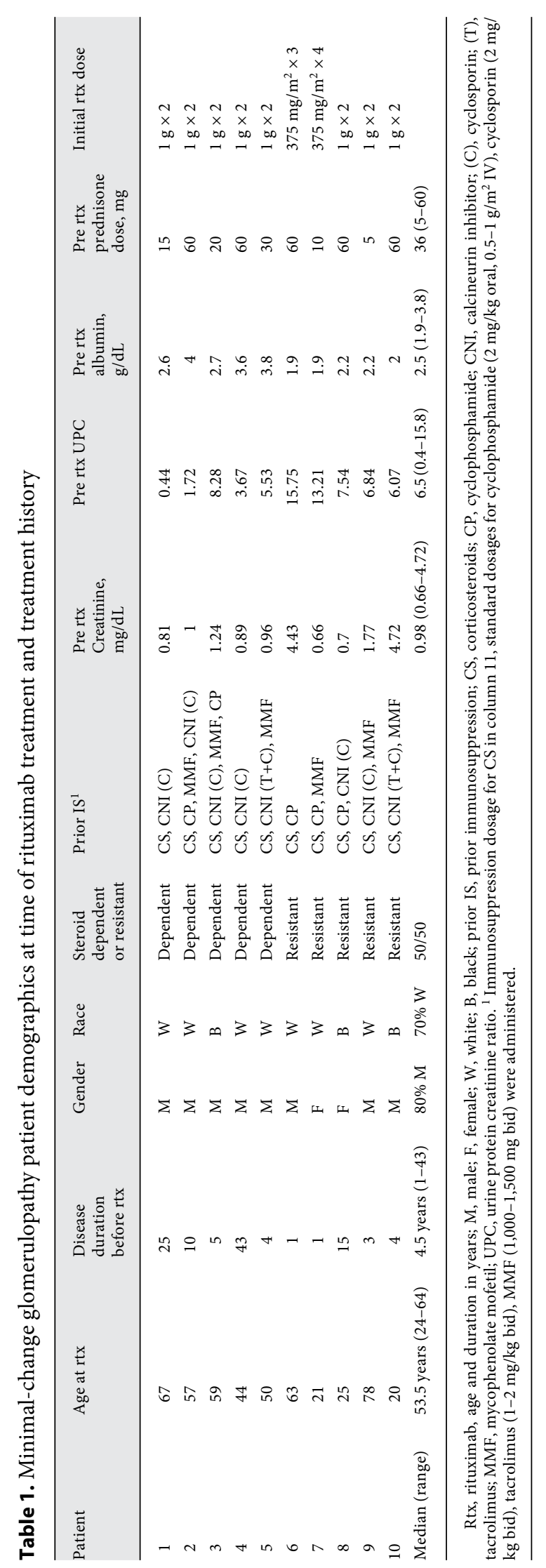


Table 2. Remission, relapse, and maintenance dosing of rituximab

\begin{tabular}{|c|c|c|c|c|c|c|}
\hline Patient & $\begin{array}{l}\text { Time from } \\
\text { induction to } \\
\text { remission, months }\end{array}$ & $\begin{array}{l}\text { Duration of } \\
\text { follow-up, } \\
\text { months }\end{array}$ & $\begin{array}{l}\text { Length of } \\
\text { remission, } \\
\text { months }\end{array}$ & $\begin{array}{l}\text { Number } \\
\text { of relapses }\end{array}$ & $\begin{array}{l}\text { Subsequent } \\
\text { doses }\end{array}$ & $\begin{array}{l}\text { Time to prednisone } \\
\text { discontinuation } \\
\text { from rituximab, } \\
\text { months }\end{array}$ \\
\hline 1 & 2 & 38 & 31 & 1 & $\begin{array}{l}8,14,22,33 \\
\text { months }\end{array}$ & 13 \\
\hline 2 & 1st 1,2 nd 1 & 80 & 1st 13,2 nd 39 & 3 & 37,52 months & 1 st 6,2 nd 2 \\
\hline 4 & 1 & 41 & 40 & 0 & None & $3-6$ \\
\hline 5 & 2 & 35 & 33 & 0 & 8 months & Never \\
\hline 6 & 2 & 46 & 44 & 0 & None & 2 \\
\hline 7 & 1st never, 2 nd 1 & 47 & 1 st 0,2 nd 22 & 1 & 25 months & 1st never, 2nd 4 \\
\hline 8 & 2 & 22 & 20 & 0 & None & 2 \\
\hline 9 & 32 & 32 & 1 & 0 & None & 0 \\
\hline
\end{tabular}

sued. However, rituximab (at $1 \mathrm{~g} \times 2$ doses) was tried 2 years later and resulted in sustained remission (22 months) and B cell depletion. The mean time from rituximab to corticosteroid discontinuation was 3.5 months (range 1-6). Nine patients were able to stay free from corticosteroid use at last point of follow-up; 1 patient initiated $<5 \mathrm{mg}$ daily for a non-related dermatological condition as needed over a year after remission with no indications of relapse of glomerular disease.

Three patients relapsed after rituximab treatment (Fig. 1; Tables 1 and 2). Patient one relapsed after 33 months in remission after a colonoscopy. Rituximab re-dosing quickly brought him back into remission. Patient 2 relapsed at 8,36 , and 74 months after receiving doses for primary induction, month 37 for relapse and month 52 for maintenance. The initial relapse was treated with rounds of cyclosporine and corticosteroids due to financial constraints. After a relapse, subsequent rituximab infusions allowed for remission until a flare 22 months after dosing. Financial limitations prohibited continued rituximab use in this patient. Patient 7 was not adequately B-cell depleted 3 months after the first rituximab induction and an attempt at maintaining her downward trend in UPC with corticosteroids and cyclophosphamide was tried. Cyclophosphamide was discontinued due to leukopenia and she was maintained on MMF, corticosteroids, and spironolactone until she became nephrotic and rituximab was again considered. After adequate dosing of 2 doses of rituximab 1,000 mg intravenously, the patient went into remission, which was maintained for 22 months.

\section{Adverse Events}

No deaths were observed based on the time of last follow-up. Non-fatal adverse events are presented in Table 3. Recurrent dose-dependent neutropenia was observed in a patient 5 months after the first dose of rituximab. However, the patient had initiated oral cyclophosphamide 1 month prior to the neutropenia due to inadequate decrease in proteinuria and the dose of cyclophosphamide had been doubled 1 week prior to neutropenia (down to 2.2). This adverse event is included in Table 3 , but is unlikely related to rituximab, as neutropenia was observed again 11 months after rituximab dosing when cyclophosphamide was increased. Two patients experienced documented upper respiratory infections that resolved without complication.

\section{Discussion}

Here, we describe the clinical course of 10 adult patients treated with rituximab for corticosteroid-dependent or -resistant minimal-change glomerulopathy. This is the first study that has examined a cohort of patients with corticosteroid-resistant disease and corticosteroiddependent or chronically relapsing courses. Following rituximab administration, all patients achieved remission from nephrotic syndrome (after a median of 2 months, range 1-6) and were successfully withdrawn from prednisone and all other immunomodulating agents (after a median of 3.5 months, range $0-13$ ). Many of these patients had a prior history of frequent and prolonged 


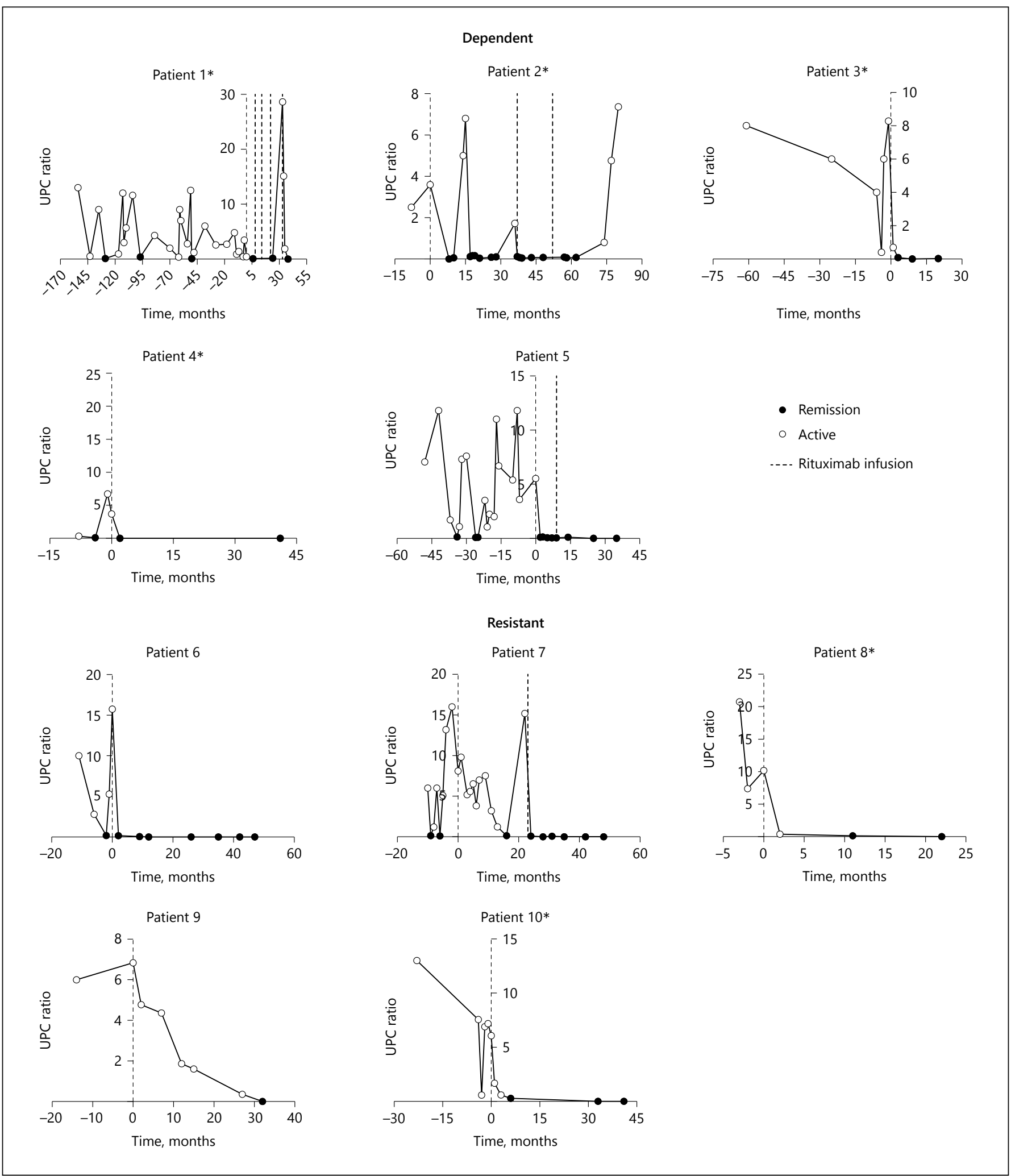

Fig. 1. Proteinuria and response to rituximab in individual patients. Patients $1-5$ are steroid-dependent, patients $6-10$ are steroid-resistant. Dashed vertical line indicates rituximab dosing, open circle indicates active disease, black closed circle indicates remission, the asterisk $\left(^{*}\right)$ denotes additional prior history without quantification of or record of proteinuria. 
Table 3. Adverse events due to rituximab treatment with confirmed B-cell depletion

\begin{tabular}{ll}
\hline $\begin{array}{l}\text { Patient } \\
\text { number }\end{array}$ & Adverse event \\
\hline 1 & None \\
2 & Upper respiratory infection (3) \\
3 & None \\
4 & Disorientation and confusion 2 weeks after infusion \\
5 & None \\
6 & None \\
7 & Neutropenia \\
8 & None \\
9 & None \\
10 & Upper respiratory infection (1) \\
\hline
\end{tabular}

courses of corticosteroids, and second-line therapies. Following treatment with rituximab, relapses were infrequent (4 relapses in 3 patients) and were successfully treated with repeated courses of rituximab (with the exception of a patient who is currently relapsing).

Insight into pathogenetic mechanisms underlying minimal-change glomerulopathy will help identify appropriate therapeutic avenues. Interestingly, rituximab can bind to human podocytes in vitro, possibly mediated through sphingomyelin phosphodiesterase acid-like $3 \mathrm{~b}$ (SMPDL-3b) protein [29], known to negatively regulate toll-like receptor signaling [30]. While podocytes do not express CD20, it is postulated that direct binding of rituximab to SMPDL-3b could prevent actin cytoskeletal changes, thereby maintaining cell integrity. It should be noted that these studies were done using samples from patients with FSGS.

Clinical research in minimal-change glomerulopathy has largely focused on children due to its high prevalence in pediatric populations. Use of rituximab for treatment of corticosteroid-dependent and corticosteroid-resistant, minimal-change glomerulopathy in children has been described in numerous published case reports, case series, and 3 randomized controlled trials in corticosteroid-dependent disease. These trials had mixed but overall favorable results, confirming therapeutic benefit $[12-14,18]$.

Data for rituximab use in adults are more limited and remain retrospective. The largest adult cohort of patients with biopsy confirmed that minimal-change glomerulopathy treated with rituximab had a $61 \%(25 / 41)$ complete clinical response with $52 \%$ of responders relapsing and had successful re-treatment with rituximab [24]. Similar recent work of 16 adults, one of whom was corticosteroid- resistant, showed full remission in $81 \%(13 / 16)$ of patients with 7 eventually relapsing after 9-28 months with a mean follow-up of 44 months [19]. Of the relapsing patients, 4 of 7 were successfully re-treated with rituximab, while the other patients were treated successfully with corticosteroids. The UNC Kidney Center cohort exhibited remission in all 10 patients with successfully treated relapses in 3 patients with rituximab. Follow up time was 40 months, similar to the longest follow-up reported in the literature [19].

Interestingly, prior to this work, studies examining the role of rituximab for minimal-change glomerulopathy have not been reported in patients in the United States and few patients with corticosteroid-resistant disease (arguably more difficult to treat than corticosteroid-dependent disease) were described. Thus, our data not only support these previous findings but also extend them to an exclusively adult US population comprised of patients with and without African ancestry with corticosteroidresistant in addition to corticosteroid-dependent disease. In our experience, rituximab is not only appears to be effective but also appears to be safe with minimal adverse events during the study period.

This study has several limitations. Selection bias was minimized in this retrospective study by including all consecutive patients treated at our center regardless of outcome. We lacked a control group, and thus outcomes cannot necessarily be attributed to rituximab and may instead reflect natural disease course. However, the patients we selected for rituximab therapy were those with prior resistant or difficult-to-treat disease, in whom spontaneous remission is unlikely. Additionally, the choice of 8 weeks of therapy to define steroid-non-responsive disease may not be appropriate for adults as they are known to have a longer response time compared to children. Finally, we did not require a repeat kidney biopsy prior to treatment for relapse; thus, disease patterns may have changed (e.g., progression to FSGS) since patients' initial diagnostic biopsy. It is also possible that patients with biopsy-proven minimal-change disease and who were resistant to corticosteroids might have had unsampled FSGS; however, all resistant patients were sampled within 1 year of rituximab and all patients responded to treatment.

This work confirms previously published observational studies of adults with minimal-change glomerulopathy supporting the benefit of rituximab therapy. All of our patients experienced a durable remission with rituximab, sparing them months to years of corticosteroid exposure. Considering these data in the context of prior reports, we 
support the notion that rituximab should be considered a valuable second-line agent for adults with relapsing corticosteroid-dependent, minimal-change glomerulopathy or as a first-line agent for corticosteroid-resistant disease. A prospective, multicenter, collaborative, randomized controlled trial is needed to assess the true efficacy as well as the optimal protocol of rituximab therapy in adult patients with corticosteroid-dependent and resistant minimal-change glomerulopathy.

\section{Disclosure Statement}

Authors have no competing interests.

\section{Support}

L.C.B. was supported by the Howard Holderness Distinguished Medical Scholars Program research fellowship grant. F.P.S. is supported by NIDDK grant T32 \#5T32DK007750-16.
WFP3, R.J.F. and P.H.N. are supported by grants from the NIDDK (P01-DK058335-14 and 1UM1-DK100845-01). WFP3 was also supported in part by NIDDK grant \# 5 F32 DK09789102 and receives translational research funding from The Broad Institute.

\section{Financial Disclosure}

None.

\section{Acknowledgments}

The authors wish to thank the patients with minimal-change glomerulopathy and all those dedicated to their care at UNC and the Glomerular Disease Collaborative Network, especially Caroline Poulton, for providing the long-standing infrastructure to make this work possible. We would like to thank Meghan Free for mentorship of L.C.B., Louis-Philippe Laurin for his initial work with these patients and Michelle O'Shaughnessey for critical review of this manuscript.

\section{References}

1 Korbet SM, Schwartz MM, Lewis EJ: Minimal-change glomerulopathy of adulthood. Am J Nephrol 1988;8:291-297.

2 Glassock RJ: Therapy of relapsing minimalchange disease in adults: a new approach? Kidney Int 2013;83:343-345.

3 Mak SK, Short CD, Mallick NP: Long-term outcome of adult-onset minimal-change nephropathy. Nephrol Dial Transplant 1996;11: 2192-2201.

4 Waldman M, Crew RJ, Valeri A, Busch J, Stokes B, Markowitz G, et al: Adult minimalchange disease: clinical characteristics, treatment, and outcomes. Clin J Am Soc Nephrol 2007;2:445-453.

5 Palmer SC, Nand K, Strippoli GF: Interventions for minimal change disease in adults with nephrotic syndrome. Cochrane Database Syst Rev 2008;1:CD001537.

6 Colattur SN, Korbet SM: Long-term outcome of adult onset idiopathic minimal change disease. Saudi J Kidney Dis Transpl 2000;11: 334-344.

7 Huang JJ, Hsu SC, Chen FF, Sung JM, Tseng CC, Wang MC: Adult-onset minimal change disease among Taiwanese: clinical features, therapeutic response, and prognosis. Am J Nephrol 2001;21:28-34.

8 Radhakrishnan J, Appel AS, Valeri A, Appel GB: The nephrotic syndrome, lipids, and risk factors for cardiovascular disease. Am J Kidney Dis 1993;22:135-142.

9 Mahmoodi BK, ten Kate MK, Waanders F, Veeger NJ, Brouwer JL, Vogt L, et al: High absolute risks and predictors of venous and arte- rial thromboembolic events in patients with nephrotic syndrome: results from a large retrospective cohort study. Circulation 2008; 117:224-230.

10 Ramachandran R, Kumar D A P, Nada R, Jha V, Gupta KL, Kohli HS: Chronic nephrotoxicity limits successful use of Tacrolimus in the management of adult steroid-dependent minimal change disease. Nephrology (Carlton) 2015;20:384-385.

11 KDIGO: Clinical practice guideline for glomerulonephritis. Kidney Int 2012;2:1-143.

12 Gilbert RD, Hulse E, Rigden S: Rituximab therapy for steroid-dependent minimal change nephrotic syndrome. Pediatr Nephrol 2006;21:1698-1700.

13 Magnasco A, Ravani P, Edefonti A, Murer L, Ghio L, Belingheri M, et al: Rituximab in children with resistant idiopathic nephrotic syndrome. J Am Soc Nephrol 2012;23:11171124.

14 Ravani P, Magnasco A, Edefonti A, Murer L, Rossi R, Ghio L, et al: Short-term effects of rituximab in children with steroid- and calcineurin-dependent nephrotic syndrome: a randomized controlled trial. Clin J Am Soc Nephrol 2011;6:1308-1315.

15 Sinha A, Bhatia D, Gulati A, Rawat M, Dinda AK, Hari P, et al: Efficacy and safety of rituximab in children with difficult-to-treat nephrotic syndrome. Nephrol Dial Transplant 2015;30:96-106.

16 Ravani P, Ponticelli A, Siciliano C, Fornoni A, Magnasco A, Sica F, et al: Rituximab is a safe and effective long-term treatment for chil- dren with steroid and calcineurin inhibitordependent idiopathic nephrotic syndrome. Kidney Int 2013;84:1025-1033.

17 Kemper MJ, Gellermann J, Habbig S, Krmar RT, Dittrich K, Jungraithmayr T, et al: Longterm follow-up after rituximab for steroiddependent idiopathic nephrotic syndrome. Nephrol Dial Transplant 2012;27:1910-1915.

18 Iijima K, Sako M, Nozu K, Mori R, Tuchida N, Kamei K, Miura K, et al: Rituximab for childhood-onset, complicated, frequently relapsing nephrotic syndrome or steroid-dependent nephrotic syndrome: a multicentre, double-blind, randomised, placebo-controlled trial. Lancet 2014;384:1273-1281.

19 Bruchfeld A, Benedek S, Hilderman M, Medin C, Snaedal-Jonsdottir S, Korkeila M: Rituximab for minimal change disease in adults: long-term follow-up. Nephrol Dial Transplant 2014;29:851-856.

20 Munyentwali H, Bouachi K, Audard V, Remy $\mathrm{P}$, Lang P, Mojaat R, et al: Rituximab is an efficient and safe treatment in adults with steroid-dependent minimal change disease. Kidney Int 2013;83:511-516.

21 Papakrivopoulou E, Shendi AM, Salama AD, Khosravi M, Connolly JO, Trompeter R: Effective treatment with Rituximab for the maintenance of remission in frequently relapsing minimal change disease. Nephrology (Carlton) 2016;21:893-900.

22 Takei T, Nitta K: Rituximab and minimal change nephrotic syndrome: a therapeutic option. Clin Exp Nephrol 2011;15: 641-647. 
23 Miyabe Y, Takei T, Iwabuchi Y, Moriyama T, Nitta K: Amelioration of the adverse effects of prednisolone by rituximab treatment in adults with steroid-dependent minimalchange nephrotic syndrome. Clin Exp Nephrol 2016;20:103-110.

24 Guitard J, Hebral AL, Fakhouri F, Joly D, Daugas E, Rivalan J, et al: Rituximab for minimal-change nephrotic syndrome in adulthood: predictive factors for response, long-term outcomes and tolerance. Nephrol Dial Transplant 2014;29:20842091.
25 Ruggenenti P, Ruggiero B, Cravedi P, Vivarelli M, Massella L, Marasa M, et al: Rituximab in steroid-dependent or frequently relapsing idiopathic nephrotic syndrome. J Am Soc Nephrol 2014;25:850-863.

26 Bagga A, Sinha A, Moudgil A: Rituximab in patients with the steroid-resistant nephrotic syndrome. N Engl J Med 2007;356:27512752.

27 Kurosu N, Sugiura H, Iwasaki C, Asamiya Y, Kojima C, Moriyama T, et al: Successful use of single-dose rituximab for the maintenance of remission in a patient with steroid-resistant nephrotic syndrome. Intern Med 2009;48: 1901-1904.
28 Yang T, Nast CC, Vo A, Jordan SC: Rapid remission of steroid and mycophenolate mofetil (mmf)-resistant minimal change nephrotic syndrome after rituximab therapy. Nephrol Dial Transplant 2008;23:377-380.

29 Fornoni A, Sageshima J, Wei C, MerscherGomez S: Rituximab targets podocytes in recurrent focal segmental glomerulosclerosis. Sci Transl Med 2011;3:85ra46.

30 Heinz LX, Baumann CL, Köberlin MS, Snijder B, Gawish R, Shui G, et al: The lipid-modifying enzyme SMPDL3B negatively regulates innate immunity. Cell Rep 2015;11:1919-1928. 